EPJ Web of Conferences $\mathrm{T}, 05025$ (2010)

DOI:10.1051/epjconf/20100प05025

(C) Owned by the authors, published by EDP Sciences, 2010

\title{
Calculations of the Triton Binding Energy with a Lorentz Boosted Nucleon-Nucleon Potential
}

\author{
H. Kamada 1,a , W. Glöckle², H. Witała ${ }^{3}$, J. Golak ${ }^{3}$, R. Skibiński ${ }^{3}$, W. N. Polyzou ${ }^{4}$, Ch. Elster ${ }^{5}$ \\ 1 Department of Physics, Faculty of Engineering, Kyushu Institute of Technology, \\ 1-1 Sensuicho Tobata, Kitakyushu 804-8550, Japan \\ 2 Institut für theoretische Physik II, Ruhr-Universität Bochum, \\ D-44780 Bochum, Germany \\ 3 M. Smoluchowski Institute of Physics, Jagiellonian University, \\ PL-30059 Kraków, Poland \\ 4 Department of Physics and Astronomy, The University of Iowa, Iowa City, IA 52242, USA \\ 5 Institute of Nuclear and Particle Physics, and Department of Physics, \\ Ohio University, Athens, Ohio 45701, USA
}

\begin{abstract}
We study the binding energy of the three-nucleon system in relativistic models that use two different relativistic treatments of the potential that are phase equivalent to realistic $\mathrm{NN}$ interactions. One is based on a unitary scale transformation that relates the non-relativistic center-of-mass Hamiltonian to the relativistic mass (rest energy) operator and the other uses a non-linear equation that relates the interaction in the relativistic mass operator to the non-relativistic interaction. In both cases Lorentz-boosted interactions are used in the relativistic Faddeev equation to solve for the three-nucleon binding energy. Using the same realistic NN potentials as input, the solution of the relativistic three-nucleon Faddeev equation for ${ }^{3} \mathrm{H}$ shows slightly less binding energy than the corresponding nonrelativistic result. The effect of the Wigner spin rotation on the binding is very small.
\end{abstract}

\section{Introduction}

For up to $300 \mathrm{MeV}$ proton energy, proton-deuteron (pd) scattering measurements have been analyzed with rigorous three-nucleon (3N) Faddeev calculations [1] based on the CD-Bonn potential [2] and the Tucson-Melbourne 3N force (3NF) [3]. Comparing theoretical calculations to the recent precise measurements of pd scattering data [4-8] indicates that theoretical predictions based on two-nucleon forces alone are not sufficient to describe the data above about $100 \mathrm{MeV}$. The minimum of the differential cross section has been discussed as the first signal of the $3 \mathrm{NF}$ effects, which are already seen below $100 \mathrm{MeV}$ [9-11]. However, presently available 3 NF's only partially improve the description of cross section data and spin observables. Since most of the cited calculations are based on the nonrelativistic formulation of the Faddeev equations [12], one needs to question if in the intermediate energy regime a Poincaré invariant formulation is required.

There are different formulations of the relativistic fewbody problem. Our calculations are based on an exact realization of the symmetry of the Poincaré group in three-

a e-mail: kamada@mns.kyutech.ac.jp nucleon quantum mechanics [13]. The mass operator (rest energy operator) consists of the relativistic kinetic energy together with two- and three-body interactions, including their boost corrections [14]. Our approach differs from a manifestly covariant scheme linked to a field theoretical approach [15].

The first attempt in solving the relativistic Faddeev equation for the three-nucleon bound state based on the second approach has been carried out in [16], resulting in a decrease of the binding energy compared to the nonrelativistic result. On the other hand, similar calculations based on the field theory approach [15] increase it. These contradictory results require more investigation.

Because the result may depend on the transformation of the nonrelativistic potential to a relativistic potential, a momentum scale transformation [17] (MST) was introduced without any additional parameters. Of course, this scale transformation method is not equivalent to the construction of a relativistic potential from a field theory. However, the scale transformation is a very useful and simple parameterization of a relativistic NN potential, which preserves the NN phase shifts exactly. Using a s-wave approximation we solved the relativistic Faddeev equation with 
Lorentz boost of the scale transformed potential $[17,18]$ and it agreed with the previous result [16].

Recently, going back to the idea of the Coester-PieperSerduke scheme (CPS), [19] we succeeded to obtain a relativistic potential numerically, and used the same method to directly construct the Lorentz boosted potential.

In the following we want to demonstrate some recent results: in Section 2 we introduce the relativistic nucleonnucleon potentials constructed by the MST and CPS methods, in Section 3 the construction of the boosted potentials is discussed, in Section 4 we give numerical results for the triton binding energy based on the Poincaré invariant Faddeev equation and in Section 5 we summarize.

\section{The Relativistic Potential}

As mentioned in the introduction, our relativistic treatment is based on the Bakamjian-Thomas framework, where the rest Hamiltonian for the three-body system consists of relativistic kinetic energies and two- and three-body interactions, including their boost corrections, which are dictated by the Poincaré algebra. The boost techniques will be discussed in the next section 3. For two-body systems realistic interactions are designed to fit scattering data. Relativistic two-body calculations must fit the same data, which means that at the two-body level the relativistic and nonrelativistic models must be phase equivalent as a function of either the center-of-momentum momentum or the energy. Differences in relativistic and non-relativistic calculations then appear first in the three-body calculations.

The usual nonrelativistic Lippmann-Schwinger equation (LS) with a potential $v$ is given in momentum space as

$$
t\left(\mathbf{p}, \mathbf{p}^{\prime} ; E\right)=v\left(\mathbf{p}, \mathbf{p}^{\prime}\right)+\int \frac{v\left(\mathbf{p}, \mathbf{p}^{\prime \prime}\right) t\left(\mathbf{p}^{\prime \prime}, \mathbf{p}^{\prime} ; E\right)}{E-p^{\prime \prime 2} / m+i \epsilon} d \mathbf{p}^{\prime \prime},
$$

where $t$ and $m$ are the t-matrix and the nucleon mass, respectively.

On the other hand, there are relations between the c.m. kinetic energy $E$ and the relative momentum $k$ for the relativistic and nonrelativistic formalism, namely

$$
\begin{aligned}
& E_{r} \equiv 2 \sqrt{m^{2}+k_{r}^{2}}-2 m, \\
& E_{n r} \equiv \frac{k_{n r}}{m} .
\end{aligned}
$$

Here the subscripts $r$ and $n r$ denote relativistic and nonrelativistic relations.

Our relativistic potential $v_{r}$ appears in the relativistic Lippmann Schwinger equation as

$$
\begin{aligned}
& t_{r}\left(\mathbf{p}, \mathbf{p}^{\prime} ; E\right)=v_{r}\left(\mathbf{p}, \mathbf{p}^{\prime}\right) \\
& +\int \frac{v_{r}\left(\mathbf{p}, \mathbf{p}^{\prime \prime}\right) t_{r}\left(\mathbf{p}^{\prime \prime}, \mathbf{p}^{\prime} ; E\right)}{2 \sqrt{m^{2}+k^{2}}-2 \sqrt{m^{2}+p^{\prime \prime}}+i \epsilon} d \mathbf{p}^{\prime \prime},
\end{aligned}
$$

where $t_{r}$ is a relativistic t-matrix. Note that in Eqs. (1) and (3) there are no subscripts ( $r$ or $n r$ ) for $E$ and $k$ (or p) before entering the next subsection. The energies $E$ in Eq.(1) and in Eq. (3) are not necessarily equal. The momenta $k$ and $\mathbf{p}$ in Eq.(1) and in Eq. (3) are not necessarily equal either.

\subsection{The Momentum Scale Transformation}

In order to build the potential $v$ one may identify the energy $E$ of Eq. (1) just as a c.m. energy $E_{e x}$ which is measured in an experiment. One may interpret [17]:

$$
E \stackrel{!}{=} E_{e x} \stackrel{!}{=} E_{r} \stackrel{!}{=} E_{n r}
$$

For this choice, $k_{r} \neq k_{n r}$.

Eq. (1) is rewritten as

$$
\begin{aligned}
& t\left(\mathbf{p}_{n r}, \mathbf{p}_{n r}^{\prime} ; E\right) \\
& =v\left(\mathbf{p}_{n r}, \mathbf{p}_{n r}^{\prime}\right)+\int \frac{v\left(\mathbf{p}_{n r}, \mathbf{p}_{n r}^{\prime \prime}\right) t\left(\mathbf{p}_{n r}^{\prime \prime}, \mathbf{p}_{n r}^{\prime \prime} ; E\right)}{k_{n r}^{\prime \prime} / m-p_{n r}^{\prime 2} / m+i \epsilon} d \mathbf{p}_{n r}^{\prime \prime} \\
& =v\left(\mathbf{p}_{n r}, \mathbf{p}_{n r}^{\prime}\right) \\
& +\int \frac{v\left(\mathbf{p}_{n r}, \mathbf{p}_{n r}^{\prime \prime}\right) t\left(\mathbf{p}_{n r}^{\prime \prime}, \mathbf{p}_{n r}^{\prime} ; E\right)}{2 \sqrt{m^{2}+k_{r}^{2}}-2 \sqrt{m^{2}+p_{r}^{\prime \prime}}+i \epsilon} d \mathbf{p}_{n r}^{\prime \prime} \\
& =v\left(\mathbf{p}_{n r}, \mathbf{p}_{n r}^{\prime}\right)+\int \frac{v\left(\mathbf{p}_{n r}, \mathbf{p}_{n r}^{\prime \prime}\right) t\left(\mathbf{p}_{n r}^{\prime \prime}, \mathbf{p}_{n r}^{\prime} ; E\right)}{2 \sqrt{m^{2}+k_{r}^{2}}-2 \sqrt{m^{2}+p_{r}^{\prime \prime 2}}+i \epsilon} \\
& \times J\left(p_{r}^{\prime \prime}\right) d \mathbf{p}_{r}^{\prime \prime},
\end{aligned}
$$

where $J$ is the Jacobian. Under the interpretation of Eq. (4) the nonrelativistic momentum $k_{n r}$ is a function of $k_{r}$,

$$
k_{n r}=k_{n r}\left(k_{r}\right)=\sqrt{2 m} \sqrt{\sqrt{m^{2}+k_{r}^{2}}-m} .
$$

One defines

$$
\begin{aligned}
& v_{r}\left(\mathbf{p}_{r}, \mathbf{p}_{r}^{\prime}\right) \equiv \frac{1}{h\left(p_{n r}\right)} v\left(\mathbf{p}_{n r}, \mathbf{p}_{n r}^{\prime}\right) \frac{1}{h\left(p_{n r}^{\prime}\right)} \\
& t_{r}\left(\mathbf{p}_{r}, \mathbf{p}_{r}^{\prime} ; E\right) \equiv \frac{1}{h\left(p_{n r}\right)} t\left(\mathbf{p}_{n r}, \mathbf{p}_{n r}^{\prime} ; E\right) \frac{1}{h\left(p_{n r}^{\prime}\right)},
\end{aligned}
$$

with

$$
h\left(p_{n r}\right) \equiv \sqrt{\left(1+\frac{p_{n r}^{2}}{2 m^{2}}\right) \sqrt{1+\frac{k_{n r}^{2}}{4 m^{2}}}} \equiv \frac{1}{\sqrt{J\left(p_{r}\right)}} .
$$

The amplitudes $t_{r}$ and $v_{r}$ are related by solving the relativistic LS equation:

$$
+\int_{r}^{t_{r}\left(\mathbf{p}_{r}, \mathbf{p}_{r}^{\prime} ; E\right)=v_{r}\left(\mathbf{p}_{r}, \mathbf{p}_{r}^{\prime}\right)} \frac{v_{r}\left(\mathbf{p}_{r}, \mathbf{p}_{r}^{\prime \prime}\right) t_{r}\left(\mathbf{p}_{r}^{\prime \prime}, \mathbf{p}_{r}^{\prime} ; E\right)}{2 \sqrt{m^{2}+k_{r}^{2}}-2 \sqrt{m^{2}+p_{r}^{\prime \prime 2}}+i \epsilon} d \mathbf{p}_{r}^{\prime \prime} .
$$

Therefore, one could identify them as relativistic amplitudes. We call Eqs. (6-8) momentum scale transformation (MST) [17].

\subsection{Coester-Pieper-Serduke Scheme}

There is another way for the identification. Instead of Eq. (4) one employs the following relation among momenta:

$$
k \stackrel{!}{=} k_{e x} \stackrel{!}{=} k_{n r} \stackrel{!}{=} k_{r}
$$


where $k_{e x}$ is the experimental momentum. In this case it is natural to add an interaction to $k^{2} / m$, so that the square of the two-body invariant mass operator becomes

$$
M^{2}=4 m^{2}+4 m\left(\frac{p^{2}}{m}+\hat{v}\right) .
$$

Because this is a function of the non-relativistic Hamiltonian, it has the same eigenfunctions as the non-relativistic Hamiltonian as a function of the relative momentum, $k$. Since the phase-shifts can be extracted from the scattering wave functions, this mass operator has the same phases shifts at the non-relativistic Hamiltonian as function of relative momentum.

The non-relativistic Lippmann-Schwinger equation still holds, Eq. (1), but in the relativistic case the interaction, the energy, and the transition operator that appear in this equation have a different interpretations than compared to the non-relativistic case.

The relation

$$
M^{2}=\left(M_{0}+\hat{v}_{r}\right)^{2}=M_{0}^{2}+4 m \hat{v}
$$

leads to the identity

$$
\left\{M_{0}, \hat{v}_{r}\right\}+\hat{v}_{r}^{2}=4 m v_{n r},
$$

which can be expressed in terms of the relative momentum operator $\hat{k}$ as

$$
4 m \hat{v}=2 \sqrt{m^{2}+\hat{k}^{2}} \hat{v}_{r}+2 \sqrt{m^{2}+\hat{k}^{2}} \hat{v}_{r}+\left(v_{r}\right)^{2}=4 m v_{n r} .
$$

Equation (14) can be expressed as the momentum space integral equation

$$
\begin{aligned}
& 4 m v\left(\mathbf{p}, \mathbf{p}^{\prime}\right)=\left(2 \sqrt{m^{2}+p^{2}}+2 \sqrt{m^{2}+p^{\prime 2}}\right) v_{r}\left(\mathbf{p}, \mathbf{p}^{\prime}\right) \\
& +\int v_{r}\left(\mathbf{p}, \mathbf{p}^{\prime \prime}\right) v_{r}\left(\mathbf{p}^{\prime \prime}, \mathbf{p}^{\prime}\right) d \mathbf{p}^{\prime \prime} .
\end{aligned}
$$

This is a nonlinear integral equation for $v_{r}$ given $v$ and can be solved by iteration [20]. The solution of $v_{r}$ is then used in Eq. (3). We call this methods Coester-Pieper-Serduke scheme (CPS).

\subsection{Relation to Realistic Potentials}

The relativistic potentials discussed here were not built directly from a relativistic Lagrangian. The nucleon-nucleon potentials were generated by requiring that they predict the experimental phase shifts. Since realistic non-relativistic potentials are constructed to fit experimental phase shifts, relativistic interactions can be constructed by requiring that they lead to the same phase shifts as the non-relativistic potentials as a function of center of momentum energy (MST) or center of momentum momentum (CPS). The MST potentials also lead to the same deuteron binding as the nonrelativistic calculation while the (CPS) potentials produce the same deuteron wave numbers as the non-relativistic calculation. The quality of $v_{r}$ obtained by each scheme was discussed in [21].
There are some realistic potentials, e.g. the Argonne V18 potential [22], which are suitable for the MST scheme because of the ansatz of Eq. (4). The CD Bonn potential [2] and the Nijmegen potential [23] are suitable to CPS because of the ansatz of Eq. (10) (See Fig.1).

The two approaches are not equivalent, but the differences at low energies are primarily due to off-shell effects.

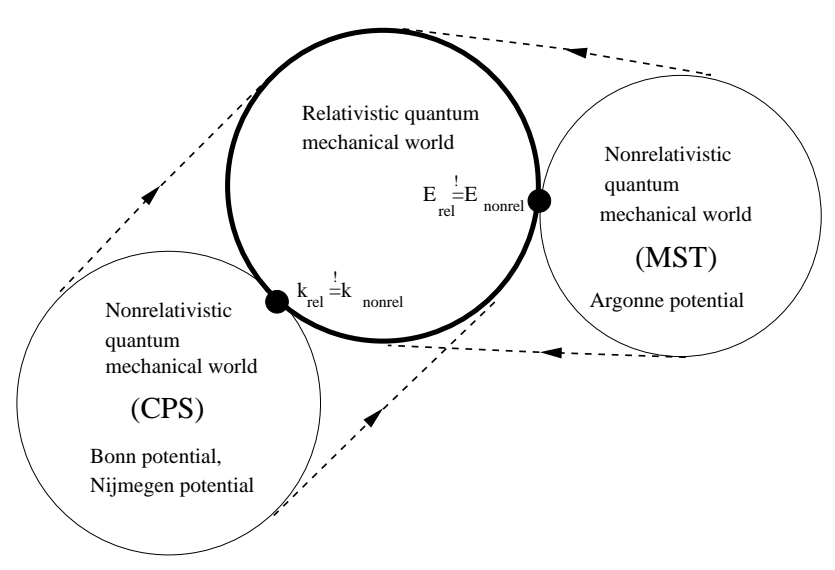

Fig. 1. Venn diagram of worlds.

\section{The Boosted Potential}

As shown in Section 2, schemes generating relativistic potential from nonrelativistic interactions are rather artificial. Compared to these schemes, the boost correction within the Bakamjian-Thomas framework is natural and unique.

Cluster properties require that the energy is additive. Because of the non-linear relations between the mass and energy in special relativity, the additivity of energies in the rest frame implies a non-linear relation between the twobody interactions in the two and three-body mass operators [13]. We call the two-body interaction in the three-body mass operator the boosted potential $v_{q}$ :

$$
\begin{aligned}
\hat{v}_{q} \equiv & \sqrt{\left(2 \sqrt{m^{2}+\hat{k}^{2}}+\hat{v}_{r}\right)^{2}+q^{2}} \\
& -\sqrt{4\left(m^{2}+\hat{k}^{2}\right)+q^{2}}
\end{aligned}
$$

where the spectator momentum $q$ in the 3-body center of mass is simultaneously the negative total momentum of the pair. In the 3-body system the momentum $q$ is operator but it behaves as c-number in the subsystem.

Using Eq. (14) this can be rewritten as potential in the CPS scheme,

$$
\hat{v}_{q}=\sqrt{4\left(m^{2}+\hat{k}^{2}+m \hat{v}\right)+q^{2}}-\sqrt{4\left(m^{2}+\hat{k}^{2}\right)+q^{2}} .
$$

Now, Eq. (17) can be rewritten as

$$
\begin{aligned}
4 m \hat{v} & =2 \sqrt{m^{2}+\hat{k}^{2}+q^{2} / 4} \hat{v}_{q}+2 \hat{v}_{q} \sqrt{m^{2}+\hat{k}^{2}+q^{2} / 4} \\
& +\left(\hat{v}_{q}\right)^{2} .
\end{aligned}
$$


This has a similar structure as Eq. (14). We have a representation in momentum space as

$$
\begin{aligned}
& 4 m v\left(\mathbf{p}, \mathbf{p}^{\prime}\right)= \\
& \left(2 \sqrt{m^{2}+p^{2}+q^{2} / 4}+2 \sqrt{m^{2}+p^{\prime 2}+q^{2} / 4}\right) v_{q}\left(\mathbf{p}, \mathbf{p}^{\prime}\right) \\
& +\int v_{q}\left(\mathbf{p}, \mathbf{p}^{\prime \prime}\right) v_{q}\left(\mathbf{p}^{\prime \prime}, \mathbf{p}^{\prime}\right) d \mathbf{p}^{\prime \prime}
\end{aligned}
$$

This is a nonlinear integral equation for $v_{q}$ in terms of $v$. Again, Eq.(19) is solved by the same iterative technique used in [20].

We would like to emphasize again that Eq. (18) is a natural extension from Eq. (14). However, Eq. (18) is not only available for the CPS scheme. In [18] the MST potential was boosted by a different way related to a Møller operator. The boost correction to the MST potential can also be calculated by this way, namely, using $v_{r}$ in Eq. (7) of MST one gets a new $v$ through Eq. (14).

\section{Triton Binding Energy}

The relativistic bound-state Faddeev equation was solved using the boosted t-matrix $t_{r}$ of Eq. (3). In Tables 1 and 2 the results for the triton binding energy using several potentials calculated based on the MST and CPS methods are displayed. The precision of the partial wave decomposition belongs to 5ch (S-wave Approximation). In the case of MST (Table 1) the results [18] show that the triton binding energies obtained from the relativistic calculation are about $400 \mathrm{keV}$ smaller compared to the ones calculated nonrelativistically. As mentioned in subsection 2.3, the Reid Soft Core potential and Argonne V18 potential can be reasonably applied in the MST scheme, but the other potential are forced to be substituted into MST method.

In the case of the CPS scheme, Table 2, the differences between the relativistic and nonrelativistic calculations are about $100 \mathrm{keV}$. The CDBonn and the Nijmgen potentials are naturally applied to the CPS scheme. This value is significantly smaller than a MSC result [18]. The reason for this overestimation of a relativistic effect on the binding energy can be attributed to a different construction of the relativistic off-shell t-matrix $t_{r}{ }^{1}$

In Table 3 we demonstrate the convergence for partial wave decomposition using CDBonn potential and the CPS scheme. In order to obtain accuracy beyond 3 digits, the total spin $j$ in the subsystem of nucleon pair, needs to be $j=4$ (34ch).

We also included the Wigner spin rotation as outlined in [26]. Thereby the Balian-Brezin method[27] in handling the permutations is quite useful. In Table 4 the triton binding energy is shown allowing charge independence breaking (CIB) [28] and Wigner spin rotations. Wigner spin rotation effects reduce the binding energy by only about $2 \mathrm{keV}$.

\footnotetext{
${ }^{1}$ In the former Proceedings [25] we would have thought that MST has a sort of defect because we need a new potential $v$ in Eq. (14).
}

Table 1. The relativistic (rel.) and nonrelativistic (nonrel) triton binding energies in MeV obtained by MST scheme from different nonrelativistic potentials. The quantity (diff.) indicates the difference between the rel. and nonrel. calculations

\begin{tabular}{lccc}
\hline potential & rel. (MST) & nonrel. & diff. \\
\hline RSC [24] & -6.59 & -7.02 & 0.43 \\
AV18 [22] & -7.23 & -7.66 & 0.43 \\
CD-Bonn [2] & -7.98 & -8.33 & 0.35 \\
Nijmegen II[23] & -7.22 & -7.65 & 0.43 \\
Nijmegen I [23] & -7.71 & -8.00 & 0.29 \\
Nijmegen93 [23] & -7.46 & -7.76 & 0.30 \\
\hline
\end{tabular}

Table 2. The relativistic (rel.) and nonrelativistic (nonrel) triton binding energies in MeV obtained by CPS scheme from different nonrelativistic potentials. The quantity (diff.) indicates the difference between the rel. and nonrel. calculations

\begin{tabular}{lccc}
\hline potential & rel. (CPS) & nonrel. & diff. \\
\hline RSC [24] & -6.97 & -7.02 & 0.05 \\
AV18 [22] & -7.59 & -7.66 & 0.07 \\
CD-Bonn [2] & -8.22 & -8.33 & 0.11 \\
Nijmegen II [23] & -7.58 & -7.65 & 0.07 \\
Nijmegen I [23] & -7.90 & -8.00 & 0.10 \\
Nijmegen93 [23] & -7.68 & -7.76 & 0.08 \\
\hline
\end{tabular}

Table 3. The theoretical predictions of the trition binding energies resulting from the solutions of the nonrelativistic (first row) and relativistic (second row) Faddeev equations as function of the number of partial waves (ch) taken into account. The note of (S) means $\mathrm{S}$-wave approximation as Tables 1 and 2. The numbers inside of braket are the maximum of the total spin in the subsystem. The last line indicates the absolute difference between the nonrelativistic and relativistic result. In the calculations only the $\mathrm{np}$ force of the CD-Bonn potential was used. Unit is in $\mathrm{MeV}$.

\begin{tabular}{lcccc}
\hline & $5 \operatorname{ch}(\mathrm{S})$ & $18 \operatorname{ch}(2)$ & $26 \operatorname{ch}(3)$ & $34 \operatorname{ch}(4)$ \\
\hline nonrel. & -8.331 & -8.220 & -8.241 & -8.247 \\
rel. & -8.219 & -8.123 & -8.143 & -8.147 \\
diff. & 0.112 & 0.107 & 0.098 & 0.100 \\
\hline
\end{tabular}

Table 4. The theoretical predictions for the relativistic and nonrelativistic triton binding energies in $\mathrm{MeV}$. All numbers are 34 channels results. The second column is the same as the last column in Table 3 . The results in the third column take charge dependence[28] into account. In addition the result of the fourth column contains Wigner spin rotation effects.

\begin{tabular}{lcccc}
\hline & np only & np+nn & Wigner rot. & diff. \\
\hline nonrel. & -8.247 & -8.005 & - & - \\
rel. & -8.147 & -7.916 & -7.914 & -0.002 \\
diff. & 0.100 & 0.089 & - & - \\
\hline
\end{tabular}




\section{Summary}

A phase-shift equivalent $2 \mathrm{~N}$ potential $v_{r}$ in the relativistic $2 \mathrm{~N}$ Schrödinger equation is related to the potential $v$ in the nonrelativistic Schrödinger equation by the momentum scale transformation scheme and the Coester-PieperSerduke scheme. The boosted potential $v_{q}$ is related to $v_{r}$ by Eq.(16). With these potentials we generate the relativistic fully-off-shell t-matrix $t_{q}$, which enters into the relativistic Faddeev equation. We solve the relativistic bound state Faddeev equation and compare the binding energy for the triton with the one obtained from a nonrelativistic calculation with the same input interaction.

In the case of the CPS scheme we find that the difference between the two calculations is only about $90 \mathrm{keV}$ including CIB, where the relativistic calculation gives a slightly reduced binding. Taking Wigner spin rotations into account in the relativistic calculation, the the binding energy is reduced by a very small amount, $2 \mathrm{keV}$, indicating that Wigner rotations of the spin have essentially no effect on the predicted value of the binding energy. Applications to the 3-body continuum are in progress. Recently [26] the formulation lined out above has been used to study the low energy Ay puzzle in neutron-deuteron scattering. In the intermediate energy regime the formulation has been applied to exclusive proton-deuteron scattering cross sections at $508 \mathrm{MeV}[29,30]$ based on a formulation of the Faddeev equations which does not employ a partial wave decomposition [31]. The approach can also be extended and applied to electromagnetic processes[32,33].

\section{Acknowledgments}

This work was partially supported by the 2008-2011 polish science funds as a research project No. N N202 077435. It was also partially supported by the Helmholtz Association through funds provided to the virtual institute "Spin and strong QCD"(VH-VI-231). The numerical calculations were performed on the IBM Regatta p690+ of the NIC in Jülich, Germany.

\section{References}

1. H. Witała et al., Phys. Rev. C 59, (1999) 3035.

2. R. Machleidt, F. Sammarruca, Y. Song, Phys. Rev. C 53, (1996) R1483.

3. S. A. Coon, W. Glöckle, Phys. Rev. C 23, (1981) 1790.

4. W. P. Abfalterer et al., Phys. Rev. Lett. 81, (1998) 57.

5. K. Sekiguchi et al., Phys. Rev. C 65, (2002) 034003.

6. K. Hatanaka et al., Phys. Rev. C 66, (2002) 044002.

7. K. Ermisch et al., Phys. Rev. C 71, (2005) 064004.

8. St. Kistryn et al., Phys. Rev. C 72, (2005) 044006.

9. K. Sagara et al., Phys. Rev. C 50, (1994)576.

10. S. Nemoto et al., Phys. Rev. C 58, (1998) 2599.

11. H. Witała et al., Phys. Rev. Lett. 81, (1998) 1183.

12. W. Glöckle et al., Phys. Rep. 274, (1996) 107.

13. F. Coester, Helv. Phys. Acta 38, (1967) 7.
14. B. Bakamjian, L. H. Thomas, Phys. Rev. 92, (1953) 1300.

15. A. Stadler, F. Gross, and M. Frank Phys. Rev. C 56, (1997) 2396.

16. W. Glöckle, T-S. H. Lee, and F. Coester, Phys. Rev. C 33, (1986) 709.

17. H. Kamada and W. Gloc̈kle, Phys. Rev. Lett. 80, (1988) 2547.

18. H. Kamada, W. Glöckle, J. Golak, Ch. Elster, Phys. Rev. C 66, (2002) 044010.

19. F. Coester, S. C. Pieper, and F. J. D. Serduke, Phys. Rev. C 11, (1975) 1.

20. H. Kamada and W. Glöckle, Phys. Lett. B655, (2007) 119.

21. T. W. Allen, G. L. Payne, and Wayne N. Polyzou, Phys. Rev. C 62, (2000) 054002.

22. R. B. Wiringa et al., Phys. Rev. C 51, (1995) 38.

23. V. G. J. Stoks et al., Phys. Rev. C 49, (1994) 2950.

24. R. V. Reid, Ann. Phys. N.Y. 50, (1968) 411.

25. H. Kamada, W. Glöckle , H. Witała, J. Golak, R. Skibiński, W. Polyzou, Ch. Elster, Mod. Phys. Lett. A 24, (2009) 804. 73, (2006) 014005.

26. H. Witała, J. Golak, W. Glöckle, H. Kamada, W. N. Polyzou, Phys. Rev. C 77, (2008) 034004.

27. R. Balian et al., Il Nuovo Cim. B2, (1969) 403.

28. H. Witała, W. Glöckle, H. Kamada, Phys. Rev. C 43, (1991) 1619.

29. T. Lin, Ch. Elster, W. N. Polyzou and W. Glöckle, Phys. Lett. B 660, (2008) 345.

30. T. Lin, Ch. Elster, W. N. Polyzou, H. Witała and W. Glöckle, Phys. Rev. C 78, (2008) 024002.

31. T. Lin, Ch. Elster, W. N. Polyzou and W. Glöckle, Phys. Rev. C 76, (2007) 014010.

32. J. Golak et al., Phys. Rep. 415, (2005) 89.

33. J. Golak, R. Skibiński, H. Witała, W. Glöckle, A. Nogga, H. Kamada, Acta Phys. Pol. B 38, (2007) 2143. 